\title{
LOW-ENERGY DEMONSTRATION ACCELERATOR (LEDA) TEST RESULTS AND PLANS ${ }^{*}$
}

\author{
H. Vernon Smith, Jr., ${ }^{\dagger}$ J. D. Schneider, and R. Sheffield \\ Los Alamos National Laboratory, Los Alamos, NM 87545, USA
}

\begin{abstract}
The LEDA 75-keV injector and 6.7-MeV RFQ have been tested with pulsed and cw proton beam currents up to 100 $\mathrm{mA}$. Several LINAC2000 papers give the results of those measurements. A follow-on experiment, to intentionally introduce and measure beam halo on the RFQ output beam, is reported in several papers at this conference (PAC2001). In this paper we summarize the LEDA RFQ commissioning results and the beam-halo measurements and we discuss future test plans for this high-current, high-average-power rf structures test bed.
\end{abstract}

\section{INTRODUCTION}

The LEDA RFQ [1] is a $100 \%$ duty factor (cw) linac that delivers $>100 \mathrm{~mA}$ of $\mathrm{H}^{+}$beam at $6.7 \mathrm{MeV}$ [2-4]. The 8-m-long, 350-MHz RFQ structure [5] accelerates the dc, $75-\mathrm{keV}, 110-\mathrm{mA} \mathrm{H} \mathrm{H}^{+}$injector beam [6] with $\sim 94 \%$ transmission. LEDA's primary objectives are to verify design codes, gain fabrication knowledge, understand beam operation, measure output beam characteristics, learn how to minimize the beam-trip frequency, and improve prediction of costs and operational availability of high-energy linac systems. One such accelerator system, a 600-MeV proton linac [7] for the accelerator-driven test facility (ADTF), is now being designed for use in the Advanced Accelerator Applications (AAA) program.

\section{RFQ COMMISSIONING}

This section summarizes the RFQ commissioning results given in [1-4] and [8-15].

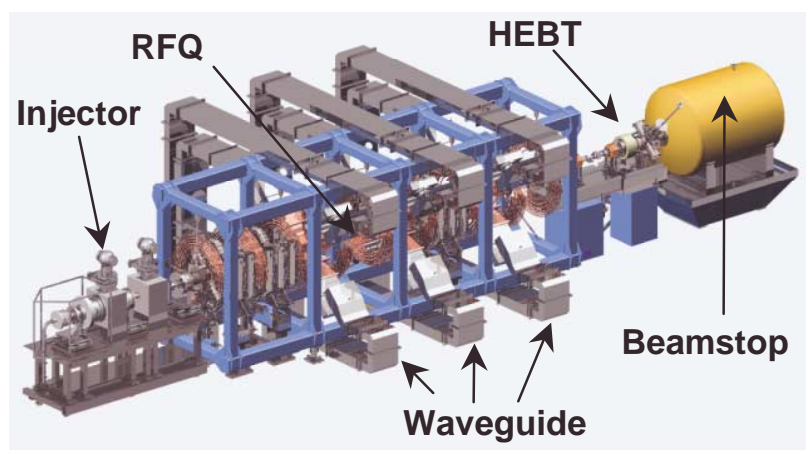

Figure 1. LEDA configuration for RFQ commissioning.

\footnotetext{
* Work supported by the US DOE, Office of Defense Programs and the Office of Nuclear Energy, Science, and Technology.

hvsmith@lanl.gov
}

\subsection{RFQ Configuration}

The accelerator configuration for beam commissioning of the LEDA RFQ is shown in Fig. 1. Major subsystems are the injector [6], RFQ [1, 2, 5, 10], high-energy beam transport (HEBT) [16], and the beamstop [17]. The injector (Fig. 2 of Ref. 3) matches the 75-keV, 110-mA dc proton beam into the RFQ. Simulations of offline injector measurements [18] indicate the RFQ input beam rms normalized emittance is $\leq 0.23 \pi \mathrm{mm}$ mrad [6]. A current modulator feeding the microwave magnetron provides beam pulsing [19] for commissioning and beam-tuning activities. The LEBT diagnostics include a pulsed-current toroid, located directly before the RFQ, which is used in determining the RFQ transmission.

A complete description of the LEDA RFQ, including the RFQ rf-field tuning procedure, resonance control, and operation with the high-power rf (HPRF) and low-level rf (LLRF) systems, is given in [1-5], [10], and the references contained therein. The function of the LEDA HEBT (Fig. 3 of Ref. 3) is to characterize the properties of the 6.7$\mathrm{MeV}, 100-\mathrm{mA}$ RFQ output beam and transport the beam with low losses to a water-shielded ogive beamstop [17]. The beamline optics consist of four quadrupole-singlet and two $\mathrm{X}-\mathrm{Y}$ steering magnets. The HEBT beam diagnostics [20] provide pulsed-beam-current and dc-beamcurrent measurements.

\subsection{RFQ Commissioning Results \& Discussion}

After modifying the injector and increasing the RFQ rf fields to $5-10 \%$ above the design values as described in [1, 2, 4, and 10], we accumulated $21 \mathrm{hr}$ of LEDA RFQ operation with $\geq 99 \mathrm{~mA}$ of $\mathrm{cw}$ output beam current and $>110 \mathrm{hr}$ with $\geq 90 \mathrm{~mA}$ of $\mathrm{cw}$ output beam current $[3,4]$.

We find that, during pulsed beam operation, the RFQ transmission drops abruptly about $100 \mu$ s into the beam pulse [4] for RFQ rf-field levels at the design value, for pulse lengths $>200 \mu \mathrm{s}$, and for RFQ output beam currents $>90 \mathrm{~mA}$. The transmission remains constant at the lower value for the duration of the pulse, including long pulses. Figure 2 shows the measured values for the total beam transmission at the start and end of a $500-\mu \mathrm{s}, 2-\mathrm{Hz}, 90-$ $\mathrm{mA}$ pulse. At the end of the pulse the total transmission deviates from the PARMTEQM prediction for 108-mA output beam current over the field-level range $88-98 \%$ of the design (Fig. 2). The total transmission at the start of the pulse follows the PARMTEQM prediction for the range $0.91-1.1$ of the design rf-field level. For output 


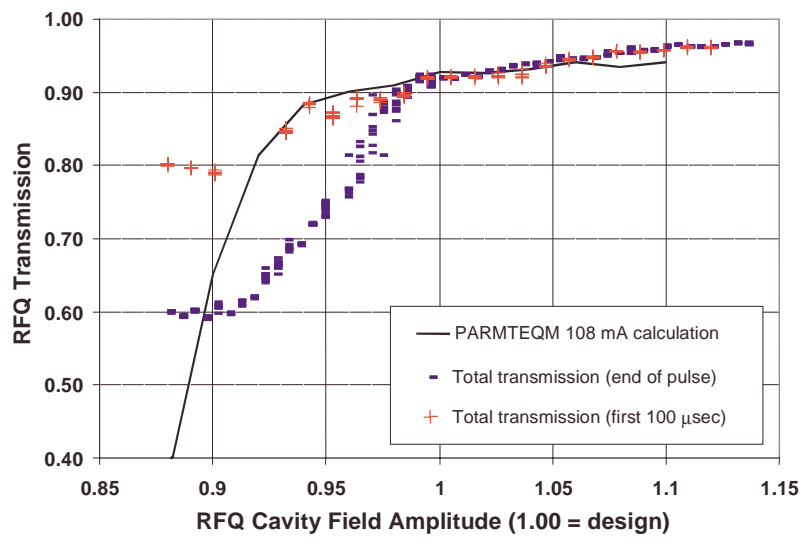

Figure 2. RFQ total beam transmission vs. rf cavity field level at the start (crosses) and end (dashes) of a 500- $\mathrm{s}$, 90-mA pulse.

beam currents $>90 \mathrm{~mA}$, e.g. $100 \mathrm{~mA}$, the RFQ transmission over the whole pulse, even cw, is increased to the design value by increasing the rf-field level to $105-110 \%$ of the design field. Both the rf-power system and the RFQ-cooling system allow this increase - the only drawback is that the RFQ requires $10-20 \%$ more input power.

The LEDA RFQ output beam emittance is determined [14,15] from quadrupole-magnet scan measurements [14]. For a 93-mA pulsed beam, the Twiss parameters are $\alpha_{x}=$ $1.8, \alpha_{y}=-2.5, \beta_{x}=36 \mathrm{~cm}, \beta_{y}=89 \mathrm{~cm}, \varepsilon_{x}=0.25 \pi \mathrm{mm}$ $\mathrm{mrad}$, and $\varepsilon_{\mathrm{y}}=0.31 \pi \mathrm{mm} \mathrm{mrad}$ (rms normalized) [14].

\section{BEAM HALO EXPERIMENT}

After RFQ commissioning, we installed a 52-magnet FODO channel [21] with which we intentionally introduce and measure beam halo [22]. The objective is to make detailed comparisons $[22,23]$ between predictions of haloformation models [24] and measured beam properties.

\subsection{Beam-Halo Channel Configuration}

A schematic drawing of the beam halo channel is shown in Fig. 3. This channel (Fig. 4) contains 52 singlet quadrupole magnets, ten $x-y$ steering magnet pairs, ten stripline beam position monitors, nine $x-y$ beam profile monitors (each unit contains a wire scanner and two halo scrapers as described in [25]), five PMT-based beam-loss monitors, and three AC current toroids [26].

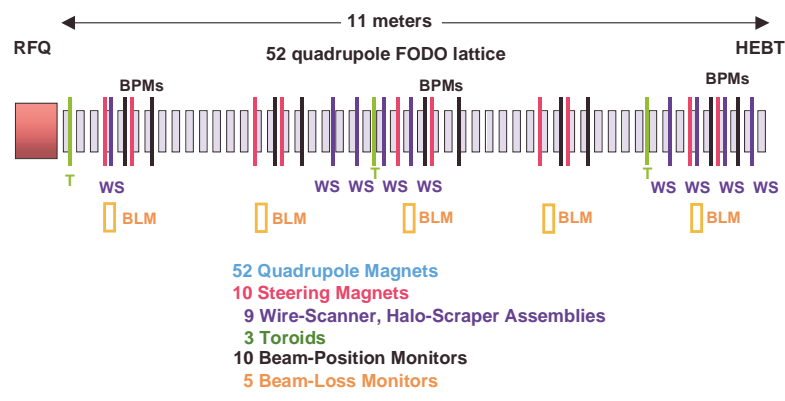

Figure 3. Schematic of the beam-halo channel. The 6.7-MeV RFQ output $\mathrm{H}^{+}$beam enters from the left and exits to the right.

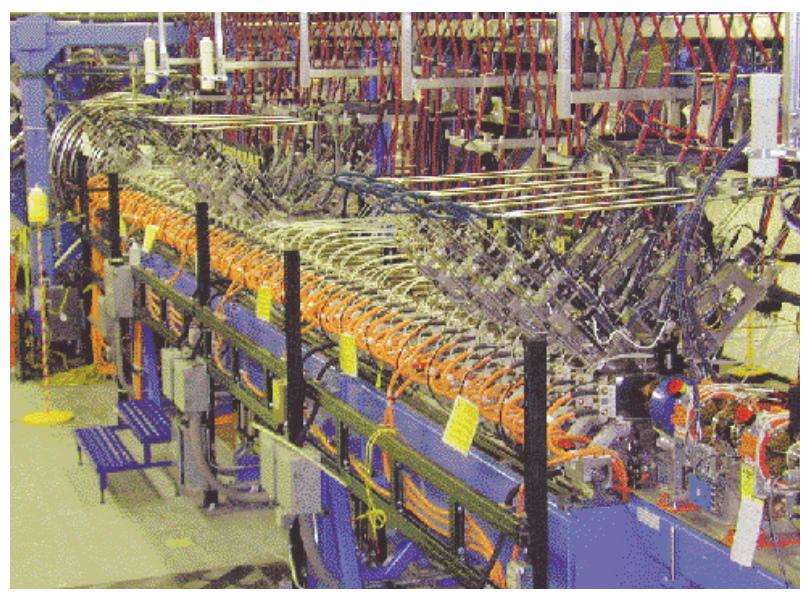

Figure 4. A halo channel photograph taken from the HEBT looking back toward the RFQ. The last four stations of wirescanner, halo-scrapers (eight units total) are visible on the right.

The HS/WS diagnostic device [25] records the beam profile with $\sim 10^{5}: 1$ signal-to-noise sensitivity (Fig. 5). The signal-to-noise sensitivity for the wire scanner is $10^{3}: 1$ and that for the halo scraper is about an additional $10^{2}: 1$. There is about a 2 -mm overlap region in which the profile is recorded with both the wire scanner and the halo scraper. The overlap region is used to "join" the data sets. A joined data set for a mismatched 75-mA beam midway down the halo channel is shown in Fig. 5 [27].

\subsection{Beam-Halo Measurements}

Measurements have been carried out for $75-\mathrm{mA}, 50$ $\mathrm{mA}$, and 16-mA RFQ output beam currents transporting down the halo channel. As detailed in [22,23], first the matched beam condition is found, then the first four quadrupole magnets are adjusted to give either breathingmode or a quadrupole-mode mismatch conditions between $\mu=0.65$ and $\mu=2$. The beam profiles are then recorded at each of the nine HS/WS locations, one just after the $\mathrm{RFQ}$, four mid-way down the halo channel, and four at the end of the halo channel. From these profiles the rms beam widths, maximum beam extent, kurtosis, and beam emittance are determined. Preliminary results, based on

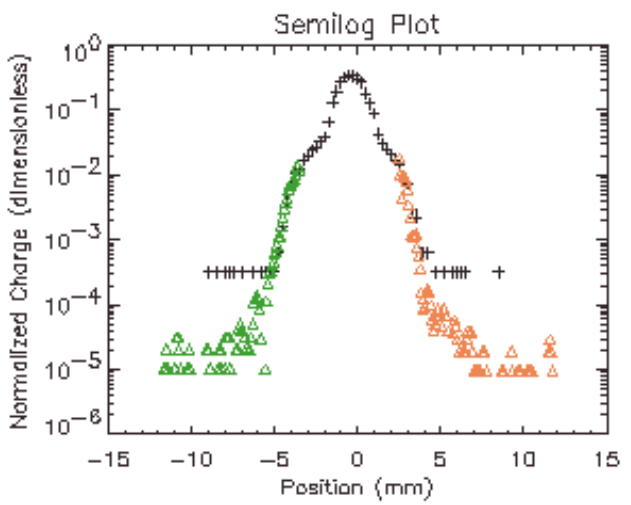

Figure 5. y beam profile midway down the FODO lattice. Wire scanner (crosses): halo scraper measurements (triangles). 


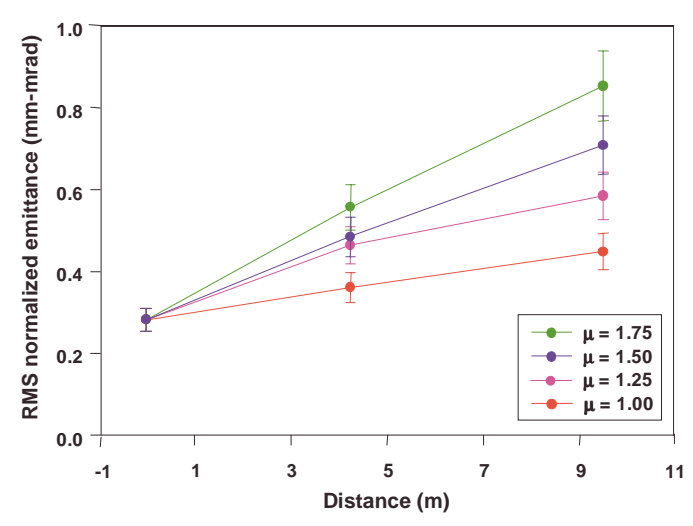

Figure 6. RMS normalized emittance in the halo channel for a 75-mA proton beam having breathing-mode mismatches.

TRACE3D analysis, for the rms-normalized emittance as a function of mismatch parameter $\mu$ at the RFQ exit (PARMTEQM calculation) and midway and at the end of the channel is shown in Fig. 6 [23]. Ten-percent error bars are shown. More halo-experiment results are given in [22], [23], and [28].

\section{FUTURE PLANS}

We initially planned to install and test two sections of coupled-cavity drift tube linac (CCDTL) structure. However, due to the success of Los Alamos tests [29] of a low-beta super-conducting spoke-resonator structure built for Shepherd et al. [30] at ANL, we now plan to test it in place of the CCDTL. This test will be of a single unit having two gaps that will accelerate the beam from 6.7 $\mathrm{MeV}$ to $\sim 7.3 \mathrm{MeV}$.

\section{SUMMARY}

The LEDA RFQ has operated with $\geq 99$-mA cw output beam for $21 \mathrm{hr}$ cumulative: it has operated $>110 \mathrm{hr}$ cumulative with $\geq 90-\mathrm{mA} \mathrm{cw}$ output beam. The RFQ output beam emittance for a 93-mA pulsed beam, determined from quadrupole-magnet-scan measurements, is $\varepsilon_{\mathrm{x}} \times \varepsilon_{\mathrm{y}}=$ $0.25 \times 0.31(\pi \mathrm{mm} \mathrm{mrad})^{2}$ [rms normalized]. After the beam-halo experiment, we plan to test a low-beta superconducting spoke-resonator structure.

\section{REFERENCES}

[1] L. M. Young, "Operations of the LEDA ResonantlyCoupled RFQ," this conf.

[2] L. M. Young, et al., "High-Power Operations of LEDA," Proc. LINAC2000 (Monterey, 21-25 August 2000) pp. 336-340. [3] H. V. Smith, Jr., et al., "Status Report on the Low-Energy Demonstration Accelerator (LEDA)," ibid., pp. 581-583.

[4] L. J. Rybarcyk, et al., "LEDA Beam Operations Milestone and Observed Beam Transmission Characteristics," ibid., pp. 584-586.

[5] D. Schrage, et al., "CW RFQ Fabrication and Engineering," Proc. LINAC98 (Chicago, 24-28 Aug. 1998), pp. 679-683.

[6] J. Sherman, et al., "Status Report on a dc 130-mA, 75-keV Proton Injector," Rev. Sci. Instrum. 69 (1998) pp. 1003-8.

[7] R. W. Garnett, et al., "Conceptual Design of a Low- $\beta$ Super- conducting Proton Linac," this conf.

[8] K. F. Johnson, et al., "Commissioning of the Low-Energy Demonstration Accelerator (LEDA) Radio-Frequency Quadrupole (RFQ)," Proc. PAC99 (New York, 29 March - 2 April 1999) pp. 3528-3530.

[9] J. D. Schneider, "Operation of the Low-Energy Demonstration Accelerator: the Proton Injector for APT," ibid., pp. 503-507.

[10] L. M. Young, et al., "Low-Energy Demonstration Accelerator (LEDA) Radio-Frequency Quadrupole (RFQ) Results," Proc. 2nd ICFA Advanced Accelerator Workshop on the Physics of High-Brightness Beams (Los Angeles, 9-12 November 1999) pp. 488-503.

[11] H. V. Smith, Jr., et al., "Update on the Commissioning of the Low-Energy Demonstration Accelerator (LEDA) RadioFrequency Quadrupole (RFQ)," ibid., pp. 466-479.

[12] H. V. Smith, Jr., et al., "Commissioning Results from the Low-Energy Demonstration Accelerator (LEDA) RadioFrequency Quadrupole (RFQ)," Proc. EPAC2000 (Vienna, 2630 June 2000) pp. 969-971.

[13] J. D. Schneider, "Overview of High-Power CW Proton Accelerators," ibid., pp. 118-122.

[14] M. E. Schulze, et al., "Beam Emittance Measurements for the Low-Energy Demonstration Accelerator Radio-Frequency Quadrupole," Proc. LINAC2000 (Monterey, 21-25 August 2000), pp. 575-577.

[15] W. P. Lysenko, et al., "Determining Phase-Space Properties of the LEDA RFQ Output Beam," ibid., pp. 809-811.

[16] W. P. Lysenko, et al., "High Energy Beam Transport Beamline for LEDA," Proc. LINAC98 (Chicago, 24-28 August 1998) pp. 496-498.

[17] T. H. Van Hagan, et al., "Design of an Ogive-Shaped Beamstop," ibid., pp. 618-620.

[18] H. V. Smith, Jr., et al., "Comparison of Beam Simulations with Measurements for the LEDA LEBT $\mathrm{H}^{+}$Beam," Proc. PAC99 (New York, 29 March - 2 April 1999) pp. 1929-31.

[19] T. Zaugg, et al., "Operation of a Microwave Proton Source in Pulsed Mode," Proc. LINAC98 (Chicago, 24-28 August 1998) pp. 893-895.

[20] J. D. Gilpatrick, et al., "Beam Diagnostics Instrumentation for the Low-Energy Demonstration Accelerator (LEDA): Commissioning and Operational Experience," Proc. EPAC2000 (Vienna, 26-30 June 2000) pp. 1717-1719.

[21] P. L. Colestock, et al., "The Beam Halo Experiment at LEDA," Proc. LINAC2000 (Monterey, 21-25 August 2000) pp. 806-808.

[22] P. L. Colestock, et al., "Measurement of Halo Generation for a Proton Beam in a FODO Channel," this conf.

[23] T. P. Wangler, et al., "Experimental Study of Proton-Beam Halo Induced by Beam Mismatch in LEDA," this conf.

[24] T. P. Wangler and K. R. Crandall, "Beam Halo in Proton Linac Beams," Proc. LINAC2000 (Monterey, 21-25 August 2000) pp. 341-345.

[25] J. D. Gilpatrick, et al., "Beam-Profile Instrumentation for Beam-Halo Measurement: Overall Description and Operation," this conf.

[26] J. D. Gilpatrick, et al., "Experience with the Low-Energy Demonstration Accelerator (LEDA) Halo Experiment Beam Instrumentation," this conf.

[27] J. H. Kamperschroer, et al., "Analysis of Data from the LEDA Wire Scanner/Halo Scraper," this conf.

[28] M. L. Schulze, et al., "Characterization of the Proton Beam from the 6.7 MeV LEDA RFQ," this conf.

[29] T. Tajima, et al., "Evaluation and Testing of a Low- $\beta$ Spoke Resonator," this conf.

[30] K. W. Shepard and T. E. Tretyakova, "Superconducting Accelerating Structures for a Multi-Beam Driver Linac for RIA," Proc. LINAC2000 (Monterey, 21-25 August 2000) pp. 920-922. 